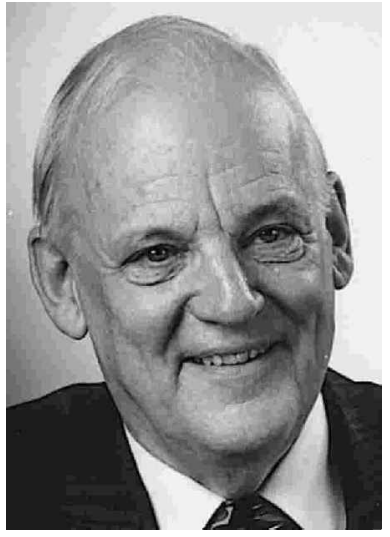

Gösta Samuelson gosta.samuelson@htu.se

\section{Wine, diet and healthy lifestyle}

$\mathrm{I}$ s wine good for you? It has been stated that moderate alcohol intake is related to improved health, and several studies during the past decade have suggested health benefits of moderate alcohol consumption. Wine drinkers have lower overall mortality rates and decreased risk for heart attacks, high blood pressure, strokes and several other diseases, according to these studies. The evidence supporting the special protective effects of wine, however, is controversial. Biological mechanisms supporting the theory have been identified, such as the positive effect of antioxidant flavonoids in wine, particularly in red wine, but recent studies have focused on confounders associated with moderate wine drinking.
The associations between different alcohol-beverage preferences and indicators of a healthy diet were investigated in a recent cross-sectional study of 2864 men and 1571 women in the south-eastern USA, most of whom were born in the mid-1940s. "The UNC Alumni Heart Study" was published in the last issue of Am J Clin Nutr 2002; 76: 466-72 by John C. Barefoot and Morten Gronbæck et al. at Duke University, North Carolina, and the Institute of Preventive Medicine in Copenhagen. The participants were homogeneous with regard to education and income. Controlling for these factors had little effect on these associations. This longitudinal study focused on cardiovascular disease risk factors, with special emphasis on psychosocial variables.

In brief, they found that wine drinkers tended to live longer and be healthier because they consumed more fruit and vegetables and had a higher dietary intake of fibre, consumed less red or fried meat, and had a lower prevalence of smoking. The nondrinkers smoked more cigarettes and consumed more fatty and unhealthy foods. They were also less likely to exercise regularly and consequently had a higher body mass index (BMI). Beer and spirit consumers tended to fall between these two groups. A limitation of the study is its reliance on self-reports; the participants may have described their lifestyles as healthier than they actually were.
The conclusion of the authors is that apparent health benefits of wine compared with other alcoholic beverages may be due to confounding effects of the dietary and other lifestyle habits of the subjects studied.

\section{Dietary products: effects on dental health}

Milk and cheese have been proven to protect against caries in rats and in situ. Several milk components, for instance casein glycomacropeptide (CGMP), reduce the adhesion of cariogenic Streptococcus mutans in situ. CGMP binds to the tooth as micelle-like structures, which do not bind bacteria. In a review article in this issue, Ingegerd Johansson gives an overview of this research field today. Many peptides in the whey fraction, i.e. proteose-peptones, protect against tooth tissue demineralization and some of them also seem to have immune-like functions. In particular, cheese that does not contain lactose appears to be of interest for further studies to identify preventive and therapeutic functions in the caries process. Selected milk components may in the future be used in children at risk, to enhance caries resistance and to combat the colonization of Streptococcus mutans. Furthermore, using molecular biology methods, components from milk or milk products could be developed for use in a saliva substitute for patients with hyposalivation and eating problems.

continued p. 115 


\section{Functional foods and health claims}

A food product bearing a health claim must be safe, and if it is consumed regularly over a long period the food item must also fit into a generally nutritious and balanced diet. Finally, and not least, the claims used in the marketing of the product must be scientifically supported.
In this issue, Nils-Georg Asp discusses health claims within the Swedish Code, which was recently extended to include "product-specific physiological claims". The first product with documentation evaluated according to the code is now on the market. Voluntary codes are also operating in the UK and the Netherlands, and European regulation is in the pipeline.

\section{New co-worker at SNF Swedish Nutrition Foundation}

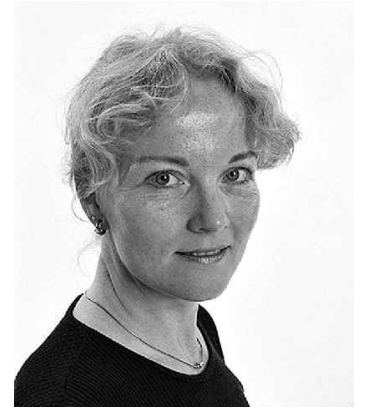

Anne Raben PhD joined the SNF Swedish Nutrition Foundation as a senior scientist in August 2002. Her PhD work at the Research Institute for Human Nutrition, Royal Veterinary and Agricultural University in Copenhagen concerned appetite and carbohydrate metabolism. She was then a senior lecturer at the same institute. Her research has focused on overweight and nutrition. This has included short- and long-term intervention studies on the effect of various diets on body weight and disease risk factors. The results have been reported in more than 70 original publications. Since 2000, Anne Raben has been the Danish editor for Scandinavian News in the Scandinavian Journal of Nutrition. 